\title{
Case Report - An Unusual Case of Vanishing White Matter Disease with Cataract and Exaggerated Stress Response
}

\author{
Forshing Lui ${ }^{1 *}$, John J Geraghty ${ }^{2}$, Alexander Jacob Nagourney ${ }^{1}$, Patrick \\ Khatchadourian ${ }^{1}$, Yosof Naseem Katiby BS ${ }^{1}$ and Anna Marika \\ Thiemann ${ }^{1}$ \\ ${ }^{1}$ California Northstate University College of Medicine, Elk Grove, California \\ ${ }^{2}$ Kaiser Permanente Medical Center, Roseville, California \\ *Corresponding Author: Forshing Lui, California Northstate University College of \\ Medicine, Elk Grove, California.
}

Received: December 28, 2021

Published: February 15, 2022

(C) All rights are reserved by Forshing Lui., et

al.

DOI: 10.31080/ASNE.2022.05.0484

\begin{abstract}
Vanishing white matter disease (VWM) is a rare inherited neurological disorder caused by mutations of the EIF2B genes (EIF2B 1 - 5). The gene is important in the initiation of translation in protein synthesis. The onset of clinical symptoms varies from infancy to adulthood. There are well-reported triggering factors for the onset of the disease as well as episodes of acute rapid decline. Apart from the antenatal or neonatal onset of the disease, the involvement of tissues or organs other than the cerebral white matter is very unusual. We are describing a case of VWM presenting with congenital cataracts at birth. Her clinical onset of leukodystrophy occurred much later during late childhood. VWM is an autosomal recessively inherited disease. However, the congenital cataract in our patient is inherited in an autosomal dominant manner. The case also demonstrated interesting and unusual triggers of vaccination and fright or stress related to separation anxiety when her neurologist went on vacation. The genetics and pathophysiology of the disease and the effects of triggers are reviewed and presented.
\end{abstract}

Keywords: Leukodystrophy; Vanishing White Matter; EIF2B Gene; Congenital Cataract

\section{Introduction}

It is easy and common for physicians to have a special connection with many of their patients. Very often, our patients trust us more than they trust their closest friends or family members. They let us know details about their personal, social, and other histories which may not be known to anyone else. Most of these special doctor-patient relationships are built on our patients' trust in us and are mostly behavioral or psychological. It is extremely unusual to have such a special connection built on the pathophysiology of the patient's physical illness.

The author is describing a case report of a patient who built a special bond and connection with him not just because of the patient's trust and psychological connection but also due to the underlying physical illness. The patient is cognitively impaired with medically refractory epilepsy due to her underlying leukodystrophy of vanishing white matter disease (VWM) [1,2]. During the last few years of her shortened life, she would present to the emergency department with a coma and more seizures resulting in hospitalization. These spells occurred whenever her neurologist (the author) went on vacation for over a week. Subsequently, the author would find the patient in the hospital waiting to be seen upon returning from his vacation.

We are reporting this case mainly because of this interesting connection which can be explained by the patient's underlying illness pathophysiologically. In addition, we will review the genetics and pathophysiology of Vanishing White Matter disease (VWM) 
$[3,4]$. VWM is an autosomal recessive inherited disease due to homozygous or double heterozygous mutations of the EIF2B genes [2]. EIF2B genes play an important role in the initiation of translation in protein synthesis. It should be expected that there would be multiorgan involvement. However, the involvement of tissues other than cerebral white matter is exceedingly uncommon. There was a report of nine patients with multiorgan involvement with neonatal or antenatal onset confirmed by genetic testing to be VWM [5]. There was one patient who had congenital cataracts among these nine cases. All these patients had homozygous or double heterozygous mutations of the EIF2B gene. Our patient has a congenital cataract at birth yet her VWM leukodystrophy did not start clinically until late childhood. In addition, her father had a congenital cataract, indicating an autosomal dominant pattern of inheritance, which is different from the usual autosomal recessive inheritance of VWM leukodystrophy.

\section{Case Report}

I first saw the patient when she was 21-years old after her regular neurologist retired. The patient was born via a normal spontaneous vaginal delivery. Her Apgar scores were six and eight. The cord clamp slipped with subsequent excessive blood loss and transient hypoxemia requiring supplemental oxygen for 24 hours. There was also transient hyperbilirubinemia. She was noted to have bilateral congenital cataracts and underwent surgery, was noted to have poor visual acuity, and developed congenital nystagmus. Furthermore, she was mildly mentally retarded with a learning disability.

Her development otherwise was relatively uneventful until at the age of 12 , when she began to complain of episodic headaches associated with confusion and slurred speech. Her first episode occurred after she received a vaccination. Her parents strongly believed that vaccination caused neurological illness. CT head at that time demonstrated ventriculomegaly and calcification in her third ventricle, so a shunt was placed (Figure 1). Her symptoms improved immediately and CSF was unremarkable except for a mildly elevated CSF protein. Day three after the procedure there was concern that the shunt had failed and it was revised but nothing was found to be wrong with the shunt. The patient continued to have recurrent episodes of screaming and confusion, so the ventriculoperitoneal (VP) shunt was revised numerous times but each time was felt to be functioning normally. At age15, the shunt was revised to a ventriculoatrial shunt. She subsequently developed both focal and generalized seizures. Her seizures were characterized by tonic extension of the right upper extremity. The seizures gradually became more frequent and resistant to anticonvulsant therapy which included lamotrigine, phenobarbital, phenytoin, topiramate, carbamazepine, and levetiracetam. There is no family history of a similar neurological disorder and the parents are not related. Her father did have a congenital cataract at a young age with normal visual acuity after cataract surgery.

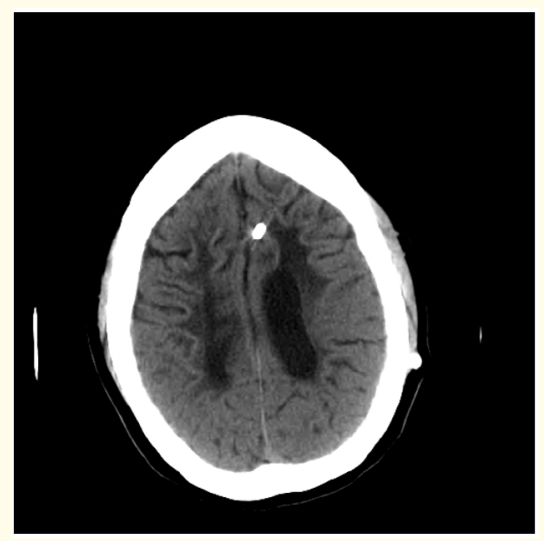

Figure 1: CT head showed ventriculomegaly with white matter hypodensity and a shunt.

When I took over her care at the age of 21 , she was ambulatory with minimal assistance. She was quite severely mentally retarded yet alert and able to follow simple commands. She had a limited vocabulary yet was able to communicate verbally. There was congenital nystagmus with bilateral optic atrophy. She had spasticity of all four extremities, generalized hyperreflexia, and bilateral extensor plantar responses.

Her brain MRI showed diffuse white matter abnormalities (Figure 2). Laboratory studies showed normal lactic acid and ammonia levels on several occasions. Very long-chain fatty acid analysis was unremarkable. CLN-1, CLN-2 and CLN-3 were negative for neuron ceroid lipofuscinosis. A subsequent skin biopsy was also negative. Acylcarnitine, homocysteine levels, and methylmalonic acid levels were normal. A wet blood smear for acanthocytes was negative and organic acid and amino acid screening was negative as well. Arylsulfatase A was negative.

After I took over her care, I recommended the placement of a vagal nerve stimulator followed by the ketogenic diet. Her seizures 
were better controlled and she became more communicative. She was able to return to special school attendance and made friends. Her parents were very happy. She continued to have seizures which were more focal affecting her left side followed by left-sided Todd's paralysis. Her parents communicated with me on the phone on a near-weekly basis.
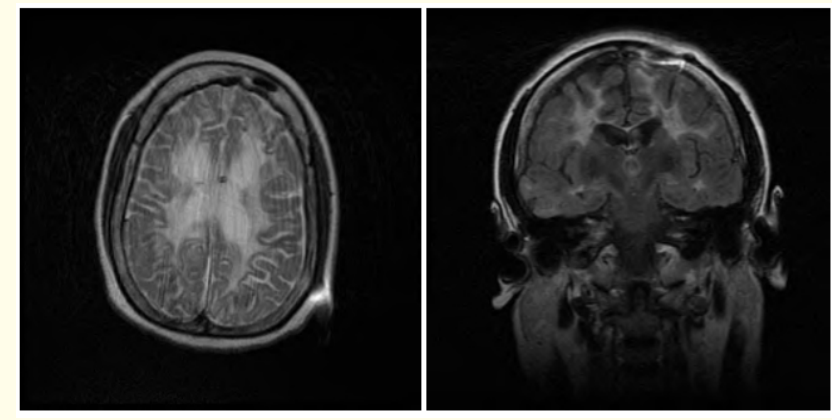

Figure 2: Axial T2 and Coronal FLAIR MRI showed diffuse white matter abnormalities. A moderately advanced stage.

Over the next few years, strange events happened. I usually had two vacations a year planned, each lasting for two weeks. When I returned from my vacation each time, the patient would have been admitted to the hospital due to status epilepticus with prolonged coma or coma without any witnessed seizures. EEG showed diffuse slowing on several occasions and occasional left-sided sharp waves. As soon as I arrived at the hospital, the patient would improve and get discharged from the hospital within a day or two. Otherwise, the patient was followed by me every three months in my office with a stable yet slowly declining neurological status. Due to the consistency between my vacations and the severe exacerbations of her seizures and coma, her parents requested me not to take any vacation longer than a week or so.

Starting from the age of about 25, there was a decline in her ability to function. She had not been ambulatory now for several years and can no longer converse. She was able to say her name and did perseverate. She requires full-time care. She started to show a right gaze preference with left hemineglect and less spontaneous movement of her left side. Her speech output also progressively decreased. Despite these neurological progressions, her pattern of status epilepticus and coma continued whenever I was on any twoweek vacation. Repeat brain MRI was performed showing progressive worsening of her white matter abnormalities (Figure 3).

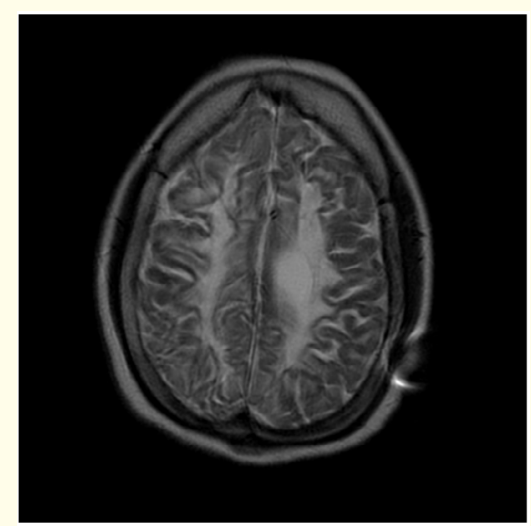

Figure 3: T2 MRI showing extensive T2 white matter disease (vanishing white matter). An advanced stage.

Finally, diagnostic genetic testing was performed confirming the diagnosis of Vanishing White Matter Leukodystrophy: Eukaryotic translation initiation factor $2 \mathrm{~B}$, subunit 5 Epsilon, 82kDa: EIF2B5 Exon sequencing: Chromosome 3q27.1; OMIM 603945. Two mutations were identified, the second known to be pathogenic for vanishing white matter disease.

The patient's neurological status continued to decline until she expired at the age of 30 . The family is grateful that we provided them with a definitive diagnosis.

\section{Discussion}

Vanishing white matter disease (VWM), is also called vanishing white matter leukodystrophy, or childhood ataxia with central hypomyelination (CACH) [6]. It is rare yet it may be one of the more common leukoencephalopathies. It is an autosomal recessively inherited disease affecting the EIF2B (Eukaryotic Initiation factor 2B) gene, EIF2B1-5 gene [2,7]. EIF2B5 gene mutation is the commonest mutation. The EIF2B gene is instrumental in protein synthesis through mRNA translation. Initiation, the first step in mRNA translation requires a set of eukaryotic initiation factors (EIF). $\mathrm{Mu}$ tation of these EIF will result in impaired mRNA translation and protein synthesis. EIF2B has also been shown to be involved in the stress response [4]. Several stress-induced kinases lead to the phosphorylation of EIF2 and convert it into a competitive inhibitor of EIF2B, which then triggers the integrated stress response. Integrated stress response dysregulation has been shown to be a causative factor in some neurodegenerative disorders. 
Typical neuropathological findings include dysmyelination, oligodendrocytes, and dystrophic astrocytes, resulting in cystic degeneration and loss of white matter volume [8].

The symptoms of VWM are progressive and, include spasticity, ataxia, motor problems, seizures, and cognitive impairment. Optic atrophy is also well reported [9]. The disease is invariably fatal yet the age of presentation and progression varies. The disease can present at any age from infancy to adulthood with childhood-onset being the most common [9]. The earlier the onset, the more severe the phenotype and the more rapid the progression. There is no gender difference in the symptoms and progression.

The onset of symptoms frequently (over $50 \%$ - natural history) occurred after a triggering event most commonly after a febrile illness [10]. Other common triggers include mild head trauma and vaccination. Acute fright (stress response) has also been reported as a triggering event. The disease course is usually one of progressive deterioration with episodes of rapid decline, which are again precipitated by infections and head trauma. Other less reported yet well-known triggers are anesthesia and acute psychological stress or fright. The pathophysiological mechanism underlying stress as a triggering factor in the onset and episodes of decline are detailed above.

Our patient demonstrated several important and interesting aspects of the disease, similar to other leukodystrophies with dysmyelinating neurological defects, VWM is caused by homozygous mutation of the EIF2B gene which is important in the initiation of translation in protein synthesis [11]. Consequently, patients with the pathological mutations will present with progressive loss of myelin and predominant involvement of long tracts including the motor, sensory, and cerebellar tracts. In addition, the initiation of protein synthesis is important in the neurons or gray matter. Disturbances will result in seizures and progressive cognitive impairment.

Our patient demonstrated several interesting clinical features that are important for our understanding of the pathophysiology and presentation of VWD and other diseases:

- $\quad$ Our patient presented with a congenital cataract at birth. Her father also had a congenital cataract. Cataract has only been described as a clinical finding of VWD in the literature with neonatal/antenatal onset associated with multiorgan involvement [5]. Our patient had congenital cataracts at birth yet her clinical VWM leukodystrophy did not start until late childhood. The vertical pattern of transmission indicates that congenital cataract is a dominantly inherited phenotype of the EIF2B mutation, which is different from the autosomal recessive mode of inheritance of her leukodystrophy. Hypomyelination and congenital cataracts (HCC) have been described in the literature [12]. It is caused by a deficiency of a membrane protein, hyccin, encoded by the gene FAM126A. We do not believe our patient suffers from HCC caused by an inherited deficiency of hyccin. The reasons include HCC is an autosomal recessively inherited condition and our patient apparently had an autosomal dominantly inherited cataract. In addition, the patient's father does not have any clinical evidence of leukodystrophy or any central nervous system problem. Unless the patient and her father's cataracts are caused by a different genetic cause which will require two rare genetic defects occurring in the same patient. Otherwise, we will have to explain that cataract in EIF2B mutation is an autosomal dominant inherited trait whereas leukodystrophy is recessively inherited. It is interesting to note that the same genetic mutation causes vanishing white matter in an autosomal recessive manner yet the congenital cataract is in a dominant fashion. In addition to myelin involvement in VWM, there have been reported cases of Brugada syndrome as an association or a trait of the disease [13]. It again exemplifies that cardiac conduction defects can also be a manifestation of EIF2B mutation.

- The onset of symptoms in our patient was triggered by vaccination [10]. It resulted in the firm belief by her parents that the vaccine caused the patient's problems, consequently leading to a strong feeling of guilt. This is why the parents were so relieved when finding out the final diagnosis. Additionally, this highlights the idea that a patient's presentation of an illness following a vaccination does not mean that the vaccine caused the illness.

- $\quad$ The last and probably the most interesting feature illustrated in this case is the effects of the stress response in triggering episodes or neurological worsening. Although the patient was already cognitively impaired when the author first took care of her, there was clearly a strong emotional connection established between them. An important reason the patient trusted her physician and his presence around her so much more was that her seizures were much better controlled after insertion of a vagal nerve stimulator, so much so that she could return to school. The fright or stress related to knowing her physician would be away for some time triggered her acute neurological 
worsening consistently over several years. This clinical feature caused her physician some significant feelings of guilt as well.

\section{Conclusion}

Vanishing White Matter disease (VWM) is an autosomal recessively inherited disease caused by mutation of the EIF2B gene which is important in the initial step of translation in protein synthesis. We reported a case of VWM with its typical clinical presentations and neuroimaging findings. In addition, we described the first case of VWM presenting with congenital cataracts which has an autosomal dominant mode of inheritance instead of the autosomal recessive mode of inheritance in VWM leukodystrophy. The significance of vaccination and fright (stress) responses are described. The patient's fright or stress response with subsequent acute neurological deterioration when her neurologist went on vacation has never been reported in the literature.

\section{Author Contributions}

Forshing Lui: planning, preparation, and drafting of the manuscript.

John J Geraghty: provision, management, and discussions about clinical cases and review of the manuscript

Yosof Naseem Katiby, Anna Marika Thiemann, Patrick Khatchadourian, and Alexander Jacob Nagourney: review and edit of the manuscript.

\section{Funding}

None.

\section{Conflict of Interest}

All authors have NO conflict of interest exist.

\section{Bibliography}

1. Bektaş G., et al. "Vanishing white matter disease with a novel EIF2B5 mutation: A 10-year follow-up". Clinical Neurology and Neurosurgery 171 (2018): 190-193.

2. Pronk JC., et al. "Vanishing white matter disease: a review with focus on its genetics". Mental Retardation and Developmental Disabilities Research Reviews 12.2 (2006): 123-128.

3. Keefe MD., et al. "Vanishing white matter disease expression of truncated EIF2B5 activates induced stress response". Elife 9 (2020): e56319.
4. Marintchev A and Ito T. "eIF2B and the Integrated Stress Response: A Structural and Mechanistic View". Biochemistry 59.13 (2020): 1299-1308.

5. Van der Knaap MS., et al. "eIF2B-related disorders: antenatal onset and involvement of multiple organs". American Journal of Human Genetics 73.5 (2003): 1199-11207.

6. Van Der Lei HD., et al. "Genotype-phenotype correlation in vanishing white matter disease”. Neurology 75.17 (2010): 1555-1559.

7. Yavuz H. "A Review of Infantile Vanishing White Matter Disease and A New Mutation". Acta Neurologica Taiwanica 26.4 (2017): 167-176.

8. Lin Y., et al. "Impaired eukaryotic translation initiation factor 2B activity specifically in oligodendrocytes reproduces the pathology of vanishing white matter disease in mice". The Journal of Neuroscience 34.36 (2014): 12182-12191.

9. Turón-Viñas E., et al. "Vanishing white matter disease in a spanish population". Journal of Central Nervous System Disease 6 (2014): 59-68.

10. Hamilton EMC., et al. "Natural History of Vanishing White Matter". Annals of Neurology 84.2 (2018): 274-288.

11. Pavitt GD and Proud CG. "Protein synthesis and its control in neuronal cells with a focus on vanishing white matter disease". Biochemical Society Transactions 37.6 (2009): 1298-1310.

12. Biancheri R., et al. "Hypomyelination and congenital cataract: broadening the clinical phenotype". Archives of Neurology 68.9 (2011): 1191-1194.

13. Güngör G., et al. "Vanishing white matter disease with different faces". Child's Nervous System 36.2 (2020): 353-361.

\section{Assets from publication with us}

- Prompt Acknowledgement after receiving the article

- Thorough Double blinded peer review

- Rapid Publication

- Issue of Publication Certificate

- High visibility of your Published work

Website: www.actascientific.com/

Submit Article: www.actascientific.com/submission.php

Email us: editor@actascientific.com

Contact us: +919182824667 